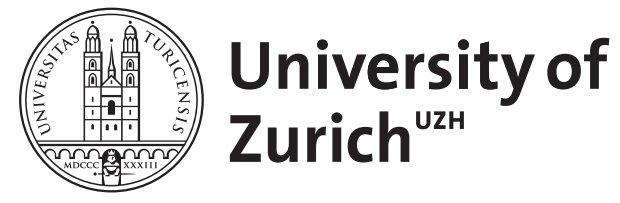

Zurich Open Repository and Archive

University of Zurich

University Library

Strickhofstrasse 39

CH-8057 Zurich

www.zora.uzh.ch

Year: 2010

\title{
Neuro-oncology, a decade of temozolomide and beyond
}

Stupp, R ; Hegi, M ; Weller, M

DOI: https://doi.org/10.1586/era.10.161

Posted at the Zurich Open Repository and Archive, University of Zurich

ZORA URL: https://doi.org/10.5167/uzh-43018

Journal Article

Accepted Version

Originally published at:

Stupp, R; Hegi, M; Weller, M (2010). Neuro-oncology, a decade of temozolomide and beyond. Expert Review of Anticancer Therapy, 10(11):1675-1677.

DOI: https://doi.org/10.1586/era.10.161 
Expert Reviews on Anticancer Therapy

$\underline{\text { Editorial }}$

\section{Neuro-Oncology, a decade of temozolomide and beyond}

It has been a decade since the regulatory approval of temozolomide, the first drug specifically developed for the treatment of malignant glioma. Initially, only a conditional approval (Food and Drug administration, USA) was granted based on a high radiological response rate in recurrent anaplastic astrocytoma, while in glioblastoma the observed responses in 2 pivotal studies were only 5 and $8 \%$, respectively [1-3]. Nevertheless, the clinical observation that disease stabilization may be clinically relevant in this disease setting led clinical investigators to pursue this agent further. In addition, the absence of better alternatives, its excellent tolerability and favorable toxicity profile have facilitated its use and investigation in a disease setting where quality of life has been a particular concern [4].

The contribution of temozolomide to the development of neuro-oncology has been far beyond its direct anti-tumor activity. The availability of a drug with (modest) single-agent activity was an important fertilizer to the field. Patients who previously had no active treatment options and who were often quickly transferred to a palliative care unit would now be seen by disease specialists. Specialized oncological care includes not only the administration of chemotherapy and regular follow-up, but also optimization of supportive measures, and revisiting the indication for steroid and antiseizure medication. Importantly, the awareness of neuro-oncology led to the creation of specialized multidisciplinary teams and clinics. Subsequent systematic academic clinical research allowed to establish combined chemoradiotherapy as the standard of care and backbone of therapy in glioblastoma [5, 6]. 
Several randomized studies in lower grade glioma are ongoing or have been recently completed. In the randomized German NOA-04 trial in anaplastic glioma treatment sequence of primary chemotherapy with radiotherapy given as salvage therapy at disease progression was compared to primary standard radiotherapy with salvage chemotherapy [7]. Both treatment strategies appear comparable. The important comparison of concomitant chemoradiotherapy versus sequential therapies is subject of the ongoing international EORTC Intergroup trial (CATNON trial, NCT \#00626990). This trial should allow to identify the relative contribution of concomitant temozolomide chemoradiotherapy and the adjuvant (maintenance) administration of this alkylating agent. While for malignant glioma with an overall still short survival combined treatment strategies with little consideration for late toxicities are justified, management of low-grade gliomas is more complex. The high variability in natural history, a median survival of over 5-7 years, (or even $>10$ years for oligodendroglioma), and the availability of only few clinical, or radiological prognostic markers are an additional challenge. Watchful waiting remains indicated for many younger patients with smaller tumors. For patients requiring therapy a randomized EORTC-NCIC trial comparing primary temozolomide chemotherapy versus standard radiotherapy has recently completed accrual (EORTC trial \#22033-26033). Correlative science (tissue submission was mandatory for central pathology review and LOH 1p determination) should allow to subsequently identify some molecular markers or profiles helping to prognosticate outcome and individualize treatment strategy.

Translational research allowed for new insights into molecular genetics and tumor genesis. Aberrant methylation of the 0-6-methylguanine-DNA methyltransferase (MGMT) gene promoter has been identified as a predictive marker for benefit from temozolomide chemotherapy in glioblastoma $[8,9]$ Ongoing studies aim at validating its assay, the optimal cutoff and its predictive value for clinical use. Molecular markers including MGMT often also carry inherent prognostic value adding to the complexity of interpretation and clinical use. Of course one does not want to withhold a potentially effective therapy from a patient, at the same time is treatment with ineffective, possibly toxic therapies 
of no benefit. Further, persisting on the use of temozolomide for all patients may prevent adequate development for more effective tailored therapies.

The (financial) success of temozolomide prompted further investment into research for brain tumors. An unprecedented number of new agents are currently in clinical trials for malignant glioma. Strategies at inhibiting VEGFmediated signaling have attracted a lot of interest and promise in malignant glioma. Impressive radiological responses and temporary clinical improvement has been demonstrated with the anti-VEGF monoclonal antibody bevacizumab in recurrent glioma $[10,11]$. Based on uncontrolled phase II trials the United States Food and Drug administration granted provisional approval for recurrent glioblastoma, while European Medicines Agency rejected the application. Most recently, cediranib, a promising VEGFR tyrosine kinase inhibitor has failed to meet its primary endpoint in a proper and well-designed phase III trial in recurrent glioblastoma. Despite persistent doubts on the quality of the antitumor effect of single-agent bevacizumab, it may be a useful agent in selected patients with large tumors and important peritumoral edema. Two large ongoing trials evaluate the benefit of bevacizumab in addition to standard chemoradiotherapy in newly diagnosed glioblastoma (AvaGlio, NCT\#00943826; RTOG0825, NCT\#00884741).

Cilengitide is a first-in-class integrin inhibitor that is currently being developed against glioblastoma in a randomized phase III trial (CENTRIC, NCT\#00689221). Based on theoretical considerations and results of a pilot phase II trial only patients with a methylated MGMT gene promoter are eligible for randomization and cilengitide therapy [12]. Despite the substantial additional challenges of upfront centralized molecular marker testing this trial is accruing well, and completion of recruitment is expected in the first quarter 2011.

Since upfront chemoradiotherapy has become standard of care of newly diagnosed glioblastoma, there is no established standard of care in recurrent disease. Enzastaurin, a protein kinase $\mathrm{C}$ inhibitor has failed to demonstrate significant single agent activity when compared with lomustine in recurrent glioblastoma [13]. Similarly the EGFR tyrosine kinase inhibitor erlotinib has not 
shown meaningful single agent activity in a randomized phase II trial [14]. Nevertheless, there remains a great need for better salvage treatments. Progress in understanding tumor biology has allowed to identify a number of key signaling pathways and processes of tumorigenesis. However, due to the redundancy of pathways and alternative signaling, inhibition at only one level may be insufficient to substantially inhibit tumor growth, and combination of several agents may be needed. However, regulatory hurdles prohibiting investigation of several non-approved agents in combination, and fierce competition among pharmaceutical companies limit the development of rational combination therapies.

Over the last decade the focus in clinical research has been on malignant glioma. The now available network of expert treatment centers and cooperative groups should allow focusing on other rare CNS malignancies, as recently demonstrated by again the German network that included over 500 patients with primary CNS lymphoma in a trial investigating the relative benefit of the addition of whole brain radiotherapy [15]. Basic research and understanding paired with early clinical observations led to exploratory clinical trials in patients with recurrent primitive neuro-ectodermal tumors (PNET)/medulloblastoma using a hedgehog inhibitor [16]. The larger cooperative group networks may want to explore this strategy in prospective randomized trials in newly diagnosed patients in combination with chemo- and radiotherapy.

The change in the field and the increased research interest is also reflected in the success of the dedicated neuro-oncology meetings by the Society of NeuroOncology (SNO) in the USA and the European Association of Neuro-Oncology (EANO), where several hundred participants are expected at their respective meetings in Montreal in November 2010 and Maastricht in September 2010. We can expect more exciting news during these meetings. 

Literature:

1. Yung WK, Prados MD, Yaya-Tur R et al. Multicenter phase II trial of temozolomide in patients with anaplastic astrocytoma or anaplastic oligoastrocytoma at first relapse. Temodal Brain Tumor Group. J Clin Oncol 1999; 17: 2762-2771.

2. Yung WK, Albright RE, Olson J et al. A phase II study of temozolomide vs. procarbazine in patients with glioblastoma multiforme at first relapse. Br J Cancer 2000; 83: 588-593.

3. Brada M, Hoang-Xuang K, Rampling R et al. Multicenter phase II trial of temozolomide in patients with glioblastoma multiforme at first relapse. Ann Oncol 2001; 12: 259-266.

4. Stupp R, Gander M, Leyvraz S, Newlands E. Current and future developments in the use of temozolomide in the treatment of brain tumors. Lancet Oncol 2001; 2: 552-560.

5. Stupp R, Mason WP, van den Bent MJ et al. Radiotherapy plus concomitant and adjuvant temozolomide for glioblastoma. N Engl J Med 2005; 352: 987-996.

6. Stupp R, Hegi ME, Mason WP et al. Effects of radiotherapy with concomitant and adjuvant temozolomide versus radiotherapy alone on survival in glioblastoma in a randomised phase III study: 5-year analysis of the EORTCNCIC trial. Lancet Oncol 2009; 10: 459-466.

7. Wick W, Hartmann C, Engel C et al. NOA-04 randomized phase III trial of sequential radiochemotherapy of anaplastic glioma with procarbazine, lomustine, and vincristine or temozolomide. J Clin Oncol 2009; 27: 5874-5880.

8. Hegi ME, Diserens AC, Gorlia T et al. MGMT gene silencing and benefit from temozolomide in glioblastoma. N Engl J Med 2005; 352: 997-1003.

9. Weller M, Stupp R, Reifenberger G et al. MGMT promoter methylation in malignant gliomas: ready for personalized medicine? Nat Rev Neurol 2010; 6: 39-51.

10. Vredenburgh JJ, Desjardins A, Herndon JE, 2nd et al. Bevacizumab plus irinotecan in recurrent glioblastoma multiforme. J Clin Oncol 2007; 25: 47224729.

11. Friedman HS, Prados MD, Wen PY et al. Bevacizumab alone and in combination with irinotecan in recurrent glioblastoma. J Clin Oncol 2009; 27: 4733-4740.

12. Stupp R, Hegi ME, Neyns B et al. Phase I/Ila study of cilengitide and temozolomide with concomitant radiotherapy followed by cilengitide and temozolomide maintenance therapy in patients with newly diagnosed glioblastoma. J Clin Oncol 2010; 28: 2712-2718.

13. Wick W, Puduvalli VK, Chamberlain MC et al. Phase III study of enzastaurin compared with lomustine in the treatment of recurrent intracranial glioblastoma. J Clin Oncol 2010; 28: 1168-1174. 
14. van den Bent M, Brandes A, Rampling R et al. Randomized phase II trial of erlotinib versus temozolomide or carmustine in recurrent glioblastoma. Report from EORTC Brain Tumor Group study 26034. J Clin Oncol 2009; 27:1268-74.

15. Thiel E, Korfel A, Martus P et al. Should whole-brain radiotherapy be considered standard of care in newly diagnosed primary central nervous system lymphoma? The G-PCNSL-SG1 randomized phase IV trial. Proc Am Soc Clin Oncol, J Clin Oncol 2010; 28: 15s (suppl; abstr \#8008\}.

16. Rudin CM, Hann CL, Laterra J et al. Treatment of medulloblastoma with hedgehog pathway inhibitor GDC-0449. N Engl J Med 2009; 361: 1173-1178. 
Author affilations:

Roger Stupp, MD

Department of Neurosurgery

Centre Hospitalier Universitaire Vaudois (CHUV) and University of Lausanne Rue du Bugnon 46

1011 Lausanne / Switzerland

Roger.Stupp@chuv.ch

Monika Hegi, PhD

Laboratory of Brain Tumor Biology and Genetics

Department of Neurosurgery

Centre Hospitalier Universitaire Vaudois (CHUV) and University of Lausanne Rue du Bugnon 46

1011 Lausanne / Switzerland

Monika.Hegi@chuv.ch

Michael Weller, MD

Department of Neurology

UniversitätsSpital Zürich

Frauenklinikstrasse 26

8091 Zürich / Switzerland

E-Mail: michael.weller@usz.ch 